Fecha de aprobación: octubre 2020

Fecha publicación: enero 2021

\section{La comunicación \\ editorial de LIJ en tiempos de redes sociales}

Nathalie Jarast ${ }^{(1)}$

Resumen: En un mundo donde la competencia con otras industrias culturales es tan grande (series, videojuegos), la comunicación y difusión del libro se vuelve central. Hoy las librerías no son el único canal de venta y, menos aún, en el mundo del libro infantil y juvenil. En este trabajo se realiza un relevamiento de los canales de estas editoriales, a través de la observación de su presencia en redes sociales y participación en ferias. Además, presenta los resultados de una encuesta (muestra piloto, exploratoria del sector), que permite obtener un panorama de las estrategias de comunicación de las editoriales de LIJ. El trabajo muestra como resultado que es fundamental incluir canales online y offline a la hora de armar una estrategia de comunicación, pero, lo más crucial para poder llegar a estas nuevas generaciones, es crear comunidades lectoras.

Palabras clave: Literatura infantil y juvenil - Comunicación editorial - Redes sociales -Comunicación digital - Libros.

[Resúmenes en inglés y portugués en la página 296]

(1) Licenciada en Letras por la Universidad de Buenos Aires, Técnica en Periodismo (TEA) y Magíster en Gestión de Contenidos por la Universidad Austral. Trabaja como periodista en diversos medios de alcance nacional y como especialista en comunicación para la industria editorial. Actualmente es responsable del área de comunicación en la editorial universitaria UNSAMedita.

\title{
Introducción
}

En un mundo donde la competencia con otras industrias culturales es tan grande (series, videojuegos), la comunicación y difusión del libro se vuelve central. Antes, se realizaba a través de la prensa (medios de comunicación tradicionales: prensa escrita, radio y TV) y mediante la recomendación del librero. Como señala un estudio de Dosdoce (2005):

Este modelo de promoción del libro tiene como base la creación de un canal unidireccional de publicación de información de la editorial (notas de prensa, 
ruedas de prensa, entrevistas con los autores, etc.) y que esperan sea publicada en los medios de comunicación tradicionales (prensa, TV y radio) con el fin de llegar a sus públicos objetivos (lectores, críticos literarios, libreros, distribuidores, otras editoriales, etc.) (p. 10).

Cabe destacar que particularmente la literatura infantil nunca tuvo demasiado espacio en los medios tradicionales; excepto por la columna “¿Qué vas a leer con tu hijo esta noche?” de Natalia Blanc en La Nación.com ${ }^{1}$ o notas esporádicas en relación a ferias, premios o acontecimientos especiales relacionados con autores. Al menos, no como la literatura para adultos que posee suplementos con reseñas destinadas a ella (Radar libros de Página 12, Ideas -anteriormente ADN Cultura- de La Nación, Revista Ñ de Clarín). Aunque sí se han de mencionar los medios especializados en LIJ como la radio Tinkuy o portales como Fundación Cuatrogatos.

Por otro lado, la lectura en la Argentina es una práctica muy asociada a lo escolar. Por ello, en este segmento, se debe considerar la comunicación con el maestro, central en la difusión y venta de los libros infantiles (tanto manuales escolares, como libros de literatura a incluir en la currícula de Lengua o Castellano). La escuela es un canal ineludible, si bien posee su propia lógica de producción, comercialización y distribución a través de promotores y compras en cantidades, así como acuerdos con las librerías cercanas a cada institución. Muchas editoriales tienen a las escuela como su principal objetivo.

Pero hoy se dan varios factores que contribuyen a un diálogo más directo entre la editorial y sus lectores y potenciales compradores. Por un lado, muchas editoriales cuentan con su propio carrito de compras, por lo que la intermediación de las librerías ya no es exclusiva. Además, hay otros modos de llegar al público. La importancia de las redes sociales entre los niños y, especialmente, los jóvenes es mayor que la recomendación del librero. Ya en 2011 Manuel Gil y Joaquín Rodríguez (2011) definían: “(...) el marketing del libro en los próximos años girará mucho más en torno a las comunidades de usuarios que al propio marketing de producto (...)” (p. 90). Según los autores, la recomendación es fundamental. Los factores que influyen en la adquisición de un libro son: “(...) el consejo de amigos o familiares, la temática (...) y el autor, en porcentajes que varían anualmente, tiene todo el sentido que las redes de recomendación cobren una relevancia fundamental y que su versión digital en forma de redes sociales basadas en afinidades jueguen un papel esencial" (p. 180).

El papel de las redes sociales es tal que se han vuelto un canal ineludible en cualquier estrategia de comunicación y marketing editorial. Se podría plantear la pregunta de si las redes no constituyen una competencia más a la lectura de libros. "Las redes no son competencia si los editores las usamos para acercar lectores a los libros; al contrario, son una herramienta de difusión", responde Trinidad Vergara, editora y creadora del sello V\&R de literatura juvenil (Conde, 2018).

Además, la participación de las editoriales en estas comunidades les ha permitido incorporar a los usuarios como recomendadores legítimos de sus productos. Méndez (2012) sostiene: "Hoy en día, blogs, Facebook y Twitter permiten un amplio acceso a las reseñas y recomendaciones 'de boca en boca.' Este fenómeno se ha profundizado en los últimos años con la aparición de los booktubers y bookgrammers que recomiendan libros en las 
redes y son convocados por las mismas editoriales para difundir sus títulos y/o publicar su propio libro. Néstor García Canclini (2015) sostiene que los booktubers, así como los libroclubes, son los nuevos mediadores entre los lectores y los libros. "Los booktubers fueron apareciendo como incitadores a leer $\mathrm{y}$, al obtener alto número de seguidores, se volvieron atractivos mediadores publicitarios para editoriales que los contratan" (García Canclini, et.al, 2015, p. 14).

Sin embargo, en la industria argentina su adopción no fue tan rápida como en otros países. Carola Martínez (2017), en el Anuario Iberoamericano sobre el libro infantil y juvenil, explica: "las editoriales argentinas tardíamente incorporaron a los bloggers y los booktubers como su focus group enviándoles novedades y sumando sus opiniones a las decisiones editoriales. Por su parte, los booktubers se han ido 'profesionalizando' y creciendo en el tema" (p. 86). Estos actores, muy activos en las redes sociales, están cambiando por completo la forma en que trabajan las editoriales destinadas a jóvenes.

\section{Una primera aproximación}

Para tener un panorama general de la comunicación de las editoriales de LIJ en la Argentina se realizó un análisis cuantitativo y cualitativo del segmento. A través de la observación y el análisis documental de las editoriales registradas en la Cámara Argentina del Libro (CAL) y en el Catálogo de editoriales independientes del Gobierno de la Ciudad de Buenos Aires, se creó un listado de editoriales que publican literatura infantil y juvenil ${ }^{2}$ y se relevaron las redes sociales en las que aparecen, su página web y su presencia en la Feria Internacional del Libro, esto último, mediante una búsqueda en su catálogo ${ }^{3}$.

Se realizó también una encuesta cerrada (véase Anexo 1), mediante Google Forms ${ }^{4}$, para terminar de construir el panorama de las estrategias de comunicación de las editoriales ${ }^{5}$. Se envió por mail a los responsables de cada editorial y fue respondida solo por 16 editoriales (véase Anexo 2). Se considera una muestra piloto, exploratoria del sector, en la que se buscó representatividad de los diversos tipos de editoriales, ya que participan sellos de distinta envergadura (chicos, medianos y grandes; independientes, de capitales extranjeros y de grandes grupos multinacionales).

\section{¿En qué redes sociales participan?}

Se observa que de las 69 editoriales relevadas, 62 tienen una página en Facebook (representa el $89 \%$ de las editoriales analizadas; véase Tabla 1). No obstante, en el caso de editoriales que publican diversas temáticas y no sólo LIJ, no todas tienen un perfil propio para el sello infantil. Es el caso de Planeta, Fondo de Cultura Económica y en editoriales pequeñas como Ediciones 36 o Editorial Camino al Sur. No obstante, en estas últimas, el sello infantil no se constituye como marca, como sí lo hace en los grandes grupos. Esto se diferencia de otras editoriales más pequeñas cuyo sector infantil tiene entidad propia en 
redes como Pípala (Adriana Hidalgo) y Uranito (Ediciones Urano Argentina). Cabe destacar que Penguin Random House, que abandonó las redes autónomas de Alfaguara infantil y juvenil cuando el sello pasó al grupo, conjuga todos sus sellos en las redes y portal del grupo “Me Gusta Leer". Sin embargo, en Argentina posee un canal especial destinado a LIJ (@novelasparachicxs), orientado fundamentalmente a jóvenes, aunque también hay algunos libros para más chicos, presente en Facebook y en Instagram. Se incluyen por esta vía textos y autores comerciales mainstream como Jojo Moyes y Tiffany Calligaris. Además, en Facebook, Alfaguara infantil posee la cuenta @librosquecuentan donde se publican sus novedades.

\section{9 editoriales}

80

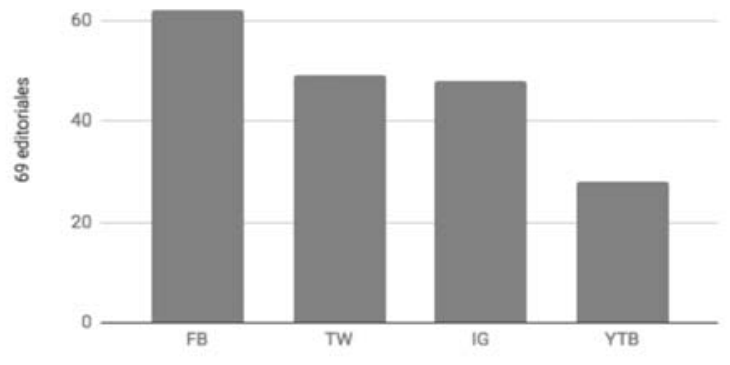

Tabla 1. Presencia en redes sociales de las editoriales de LIJ argentinas.

Esta utilización por parte de las editoriales correlaciona con el hecho de que Facebook es la red social más masiva (el 64,4\% de la población tiene una cuenta) (SinCA, 2017) y que fue una de las primeras redes sociales en adoptarse en nuestro país.

Respecto a Twitter, el 71\% de las editoriales tiene un perfil. No obstante, en esta red se publica con menor frecuencia y varias editoriales tienen este canal desactualizado. Por ejemplo, Calibroscopio y Ediciones Del Eclipse twittearon por última vez en 2016, mientras que editorial Betina lo hizo en 2015. En algunos casos, se repiten los mismos contenidos que en Facebook, mientras que en otros, se difunden noticias, contenidos diferentes o se hace cobertura de ciertas actividades. Cabe destacar que muchas editoriales no poseen un canal exclusivo para sus libros de LIJ, por lo que se encuentra información intercalada en sus redes de libros para adultos y para niños como en La marca editora o Fondo de Cultura Económica.

Prácticamente el mismo porcentaje (69\%) corresponde a Instagram, si bien esta red social está en auge en los últimos tiempos, especialmente en este público. En Argentina, los usuarios consumen más Instagram que Twitter (27,3\% y 13,2\%, según la Encuesta de 
Consumos Culturales). Un estudio de Carrier y Asociados (2017) afirma que "Twitter e Instagram reciben, también, un mayor interés de parte de los menores de 33 años (millennials y centennials)". Por lo que es central para las editoriales, hacer mayor foco en esta red. En cuanto a los contenidos publicados, funciona como Twitter. Muchas replican los contenidos de Facebook y otras, pocas, generan contenidos exclusivos para esta red.

En las encuestas, se preguntó a las 16 editoriales en qué redes estaban y con qué frecuencia publicaban contenidos. Casi todas utilizan Facebook, excepto Editorial Del Árbol. Esto correlaciona con lo observado en la primera etapa de documentación. Cabe recordar aquí, que esta red es más utilizada por los adultos y que la compra de literatura infantil suele estar atravesada por un mediador (padre/madre, docentes, bibliotecarios). Este no es el caso de los jóvenes, que deciden ellos mismos sus lecturas, en su mayoría, gracias a la influencia de sus pares. Estos últimos se encuentran más en otras redes como Instagram.

Es por eso que se incluyó en las preguntas si poseían Instagram, Twitter, Youtube, Snapchat y Whatsapp. Si bien las dos últimas conllevan una lógica más de mensajería instantánea, son las más utilizadas entre los jóvenes. La mayoría también utiliza Twitter (75\%) e Instagram $(81.25 \%)$. Esto refuerza la noción presentada en la observación documental y relevada por la Encuesta de consumos Culturales (SInCA, 2017) de que Facebook e Instagram son las redes principales en este momento. Aunque, Twitter aún mantiene un lugar preponderante en las estrategias.

En relación a Whatsapp, siete editoriales aseguran utilizar este canal de comunicación. Cinco son medianas y grandes editoriales y sólo dos, pequeñas. Cabría preguntarse si utilizan una estrategia específica en Whatsapp o sólo como una vía de contacto meramente instrumental con clientes. Por ejemplo, ¿realizan una campaña de envío masivo de invitaciones a una presentación o sólo se utiliza para responder consultas tras una compra en el e-commerce?

Es curioso que ninguna editorial utiliza Snapchat en su estrategia. Según la Encuesta de consumos culturales (SInCA, 2017, p. 33), 6.3\% de los encuestados utilizan esta red social. Por lo que es un camino interesante de exploración para las editoriales, aunque requiere de un compromiso mayor con el contenido a publicar, dado que los "posteos" de snapchat desaparecen a las 24 horas.

Respecto a la frecuencia de contenidos, diez editoriales afirman publicar diariamente contenidos en sus redes, tres semanalmente y una mensualmente (Niño Editor), mientras que dos lo hacen sólo cuando hay un nuevo lanzamiento (Lúdico y Editorial del Árbol). Las tres últimas que postean sólo ocasionalmente -una vez por mes o por un nuevo libro-son pequeñas editoriales y no poseen responsable de comunicación en su empresa. Por lo que, es comprensible que no haya una estrategia de contenidos y estos sólo se realicen a demanda. Sin embargo, algunas logran publicar semanalmente, aún sin una persona destinada a comunicación (La Brujita de Papel). Las otras dos restantes que publican semanalmente, una tiene alguien de comunicación, pero no un community manager (Arte a Babor).

Aquí cabe señalar que buenas prácticas de redes sociales indican que las páginas de Facebook e Instagram deben mantenerse activas con contenido novedoso diariamente. Facebook $^{6}$ recomienda: "Publicar en la página de tu empresa es una excelente forma de que tus clientes y fans se enteren de las novedades que ocurren en ella. Mantén el interés de los clientes y consigue que interactúen con información sobre el sector, actualizaciones de 
productos, notificaciones de eventos y mucho más". Y esto no sólo es relevante para que el público no pierda interés, sino también para el propio algoritmo que muestra más las páginas activas (con publicaciones frecuentes).

Por otro lado, desde el plano editorial, trabajar la comunicación de un nuevo libro sólo en el lanzamiento tampoco es una práctica recomendada. A diferencia de la prensa, donde se trabaja con tiempos más cortos, las redes sociales permiten anticipar la información incluso meses antes, y jugar con esos anticipos. Lionel Teti (2018), editor de Puck (el sello juvenil de Urano), recomienda comenzar con la promoción del libro en redes tres meses antes de que salga la novela, para generar expectativa y repercusión. Las redes sociales no sirven sólo para promocionar un libro, sino también para posicionar un autor y al sello que lo publica. Por ende, la comunicación con la audiencia debe ser asidua y relevante. Asimismo, el contenido no es exclusivamente de venta, sino que en muchos casos, se crean contenidos para mejorar la experiencia lectora.

En relación a la utilización de anuncios en redes sociales, sólo hay dos editoriales que no los realizan (Chirimbote y Maizal). El resto, generalmente pauta en Facebook y/o Instagram. Estas redes, permiten hacer anuncios para captar fans y seguidores, para obtener mayor alcance de una publicación (que se muestre a más personas) o mayor interacción (que las personas pongan me gusta, comenten). También permite hacer anuncios que lleven a un sitio web e incluso, con la configuración adecuada, es posible hacer anuncios de rettargeting (personas que visitaron la web, luego ven un anuncio sobre eso que vieron en sus redes). Penguin Random House también anuncia en Youtube y Google Adwords (para aparecer en primera posición en la búsqueda en Google). Esta editorial claramente cuenta con un presupuesto mayor que las otras para marketing y comunicación.

La ventaja de pautar en redes es que permite segmentar de forma muy detallada el público, además de, a través de las estadísticas, conocer a la audiencia, no sólo demográficamente, sino también por sus gustos e intereses. Al respecto, dicen Joan Van Tassel y Lisa PoeHowfield (2010): "Algunos sectores, como las imágenes en movimiento, la televisión y la radio, aprenden mucho acerca de las audiencias objetivo, pero poco sobre los consumidores individuales. Otros, como los periódicos, las revistas y, especialmente, los comercializadores de Internet, saben mucho acerca de las personas" (p. 205) ${ }^{7}$. Conocer a la audiencia es fundamental para poder participar de las conversaciones sociales que se dan en la web. Esto es crucial, en el público de YA, ya que ellos están conectados 24/7 y vuelcan todas sus opiniones y lecturas en la red. Por ello, muchas casas editoriales toman en cuenta sus comentarios y posteos a la hora de la planificación editorial: desde encontrar nuevos autores, la selección de una tapa, la relación entre la traducción y el libro en idioma original, como sobre los contenidos.

\section{El fenómeno Youtube}

En la etapa de documentación se observó que la red social menos explorada por las editoriales es Youtube (40\%). Esto es paradójico, por un lado, frente a la cada vez mayor utilización de booktrailers y, por otro, ante el auge de los youtubers en este segmento. 
Por un lado, el booktrailer es una suerte de resumen del libro, realizada en formato audiovisual. Según Tabernero-Sala (2013), "Se define como un modo de promoción que tiene que ver con un lector, el del siglo XXI, que se desenvuelve de una forma natural en las redes sociales y recibe la información a través de soportes multimedia que integran palabra, imagen y sonido de naturaleza hipertextual". Es por lo tanto, llamativo que más de la mitad de las editoriales registradas no cuenten con un canal de Youtube. De ellas, la mayoría pertenecen a editoriales independientes. Por el contrario, las 28 editoriales que sí cuentan con este canal incluyen a los grandes grupos, las editoriales de capitales extranjeros como Océano, Fondo de Cultura Económica, Edelvives y algunas independientes y pequeñas como Ciccus, Editorial Chirimbote, La Brujita de Papel, Pípala, entre otras. Además de booktrailers, en los canales observados se encuentran coberturas de presentaciones y entrevistas. De hecho, son muy pocas las que publican booktrailers.

En este sentido, las encuestas revelaron que sólo el 37.5\% utiliza Youtube. ¿Quiénes son las editoriales que usan este último canal? Norma, Eduvim, SM Argentina, Niño Editor, Guadal y Penguin Random House. Es decir, de las seis, cinco pertenecen a medianas y grandes editoriales. Estas, además, realizan acciones con influencers, mientras que la editorial pequeña, no.

Nuevamente, se menciona aquí la importancia de este canal para el público joven. Según la Encuesta de consumos culturales (2017): "La visualización online a través de aplicaciones con abono o sitios gratuitos de internet tiene un comportamiento bastante asociado a la edad. Los que más consumen por ambas vías son los jóvenes (los adolescentes de 12 a 17 años son los grandes consumidores de YouTube)" (p. 18). Es por ello que llama la atención que estas editoriales no cuenten con un canal en Youtube. Una posible hipótesis de por qué no apuestan a este canal, es porque algunas cuentan con literatura infantil y no para adolescentes. Este es el caso de La Brujita de Papel (publica libros hasta 10 años), Lúdico y El gato de hojalata, por ejemplo. Sin embargo, otras como Abran Cancha poseen títulos a partir de 12 años y aun así no apuesta a este canal. Tabernero- Sala (2013) dice: “(...) los booktrailers vinculados a la literatura juvenil tienen que ver con los diseños materiales de los libros que promocionan, diseños relacionados con el consumo cultural de los adolescentes del momento actual (...)" (p. 212). Sin embargo, el mismo autor asegura que este modo de comunicación también es aplicable a la literatura para más chicos, especialmente en el caso del libro álbum ${ }^{8}$, dado que comparten el hecho de utilizar diferentes códigos y lenguajes. La proximidad del libro álbum y el lenguaje fílmico convierten el booktrailer en uno de los medios más adecuados para la publicitación del género.

\section{Influencers de libros}

La recomendación, especialmente si es entre pares, tiene más relevancia que cualquier otro tipo de promoción o publicidad. Al respecto, Birkner (2013) asegura: “(...) los editores deben concentrarse en el mayor motor de marketing del mercado, según los expertos: una recomendación de pares" (p. 45). El marketing de influencers, tan de moda en otras industrias, también forma parte del mundo del libro. En este sentido, la literatura juvenil 
es la que ha hecho mayor uso de esta estrategia, aunque también hay casos de literatura para adultos y niños que apuntan a influencers, en este caso, adultos. En la industria, se los denomina BBB's: bloggers, booktubers y bookstagrammers.

Youtube ha permitido la legitimización de los booktubers (acrónimo de book y youtubers como recomendadores) ${ }^{9}$. Estos son jóvenes entre 16 y 25 años que se dedican a reseñar, criticar o comentar libros en formato de video. Para Lluch (2017), que analizó youtubers de España durante varios años, "El cambio de siglo se ha inaugurado con una fuerte presencia de adolescentes y jóvenes en el mundo de la lectura como fuente de autoridad, ejerciendo tareas que tradicionalmente han realizado los adultos (críticos literarios) y legitimando un nuevo canon (en el sentido de una lista de lecturas que hay que conocer para socializarse)" (p. 32). De hecho, Penguin Random House posee en su sitio web una sección ${ }^{10}$ destinada a booktubers y bloggers que se inscriben para reseñar libros de la editorial. Al respecto, Francisco Albarello, Arri y García (2018) señalan:

Las editoriales argentinas, por ejemplo, desarrollan distintas estrategias con los booktubers: en algunos casos se contactan con ellos y les ofrecen su catálogo de novedades para que elijan qué libro reseñar, mientras que en otros casos le solicitan a los booktubers que reseñen determinados libros. Los booktubers se han transformado en los últimos años en un nuevo tipo de 'mediadores culturales' (García Canclini, Gerber Bicecci, López Ojeda, Nivón Bolán, Pérez Camacho, Pinochet Cobos y Winocur Iparraguirre, 2014) entre la industria cultural y el mercado de consumidores de libros, produciéndose de este modo una lectura de contagio entre los booktubers y sus seguidores.

Estos actores, además, se han vuelto verdaderas estrellas mediáticas en las ferias del libro en toda Latinoamérica. En este sentido, la industria editorial argentina les ha dado un espacio muy importante en la Feria Internacional del Libro de Buenos Aires (la de adultos, no la infantil), con un encuentro de booktubers internacionales, a sala colmada de jóvenes. Esta generación revolucionó la feria. "Algunos pensaban que era una moda, pero ya se cumplieron 20 años de Harry Potter y 10 de Los juegos del hambre. Eso fue un viaje de ida. Después los chicos descubrieron que querían seguir leyendo y algunos que además quería escribir o ser editores. Hoy son los actores más importantes en un mercado difícil”, dice Cristina Alemany, representante de la comisión juvenil de la Fundación El Libro (Scherer, 2018).

No es de extrañar que las editoriales hayan visto en esta comunidad un espacio clave donde publicitarse a través de una serie de acuerdos de mutuo beneficio con los booktubers. Garcés Estrada, Avitia y Ramírez (2018) explican: "La temática de esta colaboración es sencilla, las editoriales envían los libros a los booktuber y ellos las dan a conocer a sus seguidores, resaltando lo que les gustó o no del libro, incluso una recomendación negativa del libro puede llamar la atención de los usuarios" (p. 752). Pero en la actualidad, no sólo son voceros de las editoriales, sino también se constituyen como autores consagrados (o al menos, publicados en papel), gracias a ellos. Lluch (2017) sostiene: "Estos escritores han sustituido las visitas a los colegios o las entrevistas en la prensa en papel por la relación directa con sus lectores a través de los medios sociales (...)” (p. 34). El caso más emblemá- 
tico es el del chileno Germán Garmendia, cuyo libro fue uno de los más vendidos en 2017. Esto también se da fuertemente en Instagram con los bookstagramers. Pero ¿qué libros recomiendan? ¿qué editoriales utilizan influencers? Generalmente, corresponden a publicaciones de los grandes grupos o de editoriales independientes dedicadas al sector YA (young adult) como V\&R. Pero la literatura infantil no se queda atrás. Hay muchos padres/ madres/docentes que se han vuelto influencers y recomiendan libros para niños. Incluso muchos de ellos también venden libros a través de librerías virtuales o clubes de lectura como@cuentosparanina, @lecturita, @bimbambooks. A través de la encuesta realizada, se intentó vislumbrar quiénes son los actores que acceden a este tipo de marketing.

Entre las editoriales encuestadas, prácticamente la mitad dice realizar acciones con influencers: Chirimbote, Norma, Eduvim, La Editorial común, SM, Arte a Babor y Penguin Random House. Cabe señalar que dos de ellas pertenecen a los grandes grupos y otraSM- a capitales extranjeros. Esta última, puntualmente tiene un programa de recomendación de libros con BBB. Respecto al tipo de acciones, estas editoriales señalan: "Entrega de ejemplares para que comenten y recomienden", "Prensa o sorteos", "Encuentros en las ferias, envío de novedades".

Estos encuentros, generalmente en el marco de la Feria del Libro, una presentación o un desayuno o evento, son diseñados especialmente para ellos. Entre algunas acciones que se realizan con estos BBB son fundamentales las que acercan el lector al autor. Así, en palabras de Leonel Teti (2018), se utilizan estrategias como Blog tours (se entregan ejemplares a bloggeros para reseñar y para sortear, sumado a contenido exclusivo como fotos del autor, un fragmento que se dejó fuera, una respuesta del autor, etc.), Twitter Chat (una suerte de entrevista con el autor vía Twitter, donde se hacen las preguntas siguiendo un hashtag) y Google Hangout (una entrevista por videoconferencia). Norma, SM y Penguin señalan que realizan encuentros presenciales con influencers, dentro de sus estrategias.

¿Por qué es tan importante mantener a este grupo de BBB? Alonso- Arévalo y CordónGarcía (2011) responden:

Las recomendaciones en las redes sociales han desempeñado un papel fundamental en este caso y en muchos otros en los que los medios de comunicación tradicionales, el crítico convencional o los canales literarios apenas han tenido incidencia alguna. Surge la figura del influencer, esto es, la persona capaz de trasladar opiniones a miles de seguidores con gran capacidad de persuasión, gracias al crédito, a la reputación digital obtenida con sus intervenciones en Twitter, Facebook, Linkedin o cualquier otra red (p. 267).

Pero los influencers no sólo son promotores y recomendadores de libros, sino que se han convertido en jugadores que pueden cambiar la trama de una historia y otros elementos del proceso editorial. María José Ferrari, editora del Departamento Infantil y Juvenil del Grupo Planeta, asegura en una nota para La Nación (Krom, 2018): "Muchas veces los tenemos como proofreaders para que preparen informes de lectura", y señala que incluso pueden incidir en la elección de qué obras se van a publicar: "A veces traen reseñas de libros que no se editan acá, están anticipados a las series y las películas, tienen una agenda literaria y cultural muy rica, y uno trata de estar atento a lo que ellos están viendo. No 
siempre podemos responder a todos los pedidos, pero tratamos de tener la cintura para ajustarnos a sus demandas".

Muchos se han convertido en "evaluadores editoriales". Como se explica en otra nota de La Nación (Sigal, 2018), el concepto nació en el mercado anglosajón como "sensitivity readers" para evaluar los contenidos sobre temáticas que pueden generar controversia como racismo, homofobia o abuso sexual. En el país, frente al crecimiento de publicaciones sobre bullying, suicidio o depresión se recurrió a la figura de evaluador editorial. Su función principal es analizar la potencialidad comercial de los proyectos. Se eligen BBB, ya que son quienes consumen este tipo de literatura.

Por otro lado, algunas de las editoriales infantiles (no juveniles) también apuntan a influencers adultos como mommy y daddy bloggers, sucede en La Editorial Común, Arte a Babor y Penguin Random House, o perfiles de recomendación literaria como Chirimbote, Eduvim y Norma. Desde Penguin Random House también señalan realizar acciones con marcas para chicos para promover sus libros. Aquí el par no es el niño/ consumidor final, sino el mediador, que tiene la decisión de compra. En este sentido, Estelle Jobson (2001) afirma: "La comercialización a los niños implica inevitablemente la comercialización a sus padres. Los padres están ansiosos por saber exactamente qué encontrarán sus hijos en línea y es posible que deban estar convencidos de los posibles beneficios (educativos) de un sitio para niños" (p. 25) ${ }^{11}$. Es por ello, que las acciones offline, tanto para los medios tradicionales como para los lectores sigue teniendo vigencia en este terreno.

\section{Otras estrategias}

Respecto al uso del mailing, casi todas las editoriales encuestadas lo utilizan (87.5\%). Abran Cancha y Lúdico ediciones son las únicas que no registraron utilizar e-mail marketing. Se utiliza para novedades, gacetillas, invitación a eventos. Se dirige directamente al lector/ consumidor, que aportó su mail en algún momento, dado que le interesaba esa editorial o alguno de sus libros.

Van Tassel y Poe- Howfield afirman (2010): “(...) Una vez que el responsable de marketing tiene la dirección de correo electrónico, él o ella circula a otro medio: correo electrónico, que establece una relación de uno a uno con el consumidor" (p. 346). Muchas de las editoriales cuentan con un sistema de registración en sus páginas web donde un usuario se puede suscribir al newsletter o boletín de novedades, y así se va generando la base de datos de la editorial. Una buena estrategia debería incluir una segmentación de esa base, asociando el contenido de los mails a los intereses de cada uno (periodistas, público general, padres, docentes y dentro de ellos, si les interesan los libros de 0-4 o de 12-14, por ejemplo). Algunas editoriales recopilan datos en eventos también o con un mensaje al final del libro que insta a escribir a la editorial contando la opinión. Si el mail proviene de alguna de estas dos formas, es más fácil segmentar los intereses. Si no, será tarea del responsable de marketing enviar una pequeña encuesta para que el público complete sus intereses y así reciba un $10 \%$ de descuento en la siguiente compra, por ejemplo. 
Respecto a la venta a través de $e$ - commerce 10 editoriales de las encuestadas manifiestan utilizar este canal para la venta de libros. Aquí el vínculo con el comprador es directo y se elimina el intermediario de la librería. Es curioso que, a diferencia de otros parámetros, la existencia de e-commerce no está necesariamente relacionado al tamaño de la editorial. Entre las que no tienen figuran editoriales pequeñas, medianas y grandes grupos: Lúdico, Norma, Hola chicos, Editorial del Árbol, SM y Penguin Random House. Este último posee en su página un botón "Lo quiero" que permite seleccionar el libro en papel, ebook o audiolibro y luego comprarlo en tiendas de librerías como Tematika, Cúspide, Librería Santa Fe.

Más allá de los acuerdos comerciales que puedan existir, las grandes editoriales cuentan con canales de distribución mucho más aceitados. Sumado a que pueden acceder a realizar publicidad en grandes librerías con banners, mesas especiales, cartelería, etc. Es por eso, que la venta directa favorece en mayor medida a las pequeñas editoriales, ya que éstas acortan el camino hacia el lector y evitan costos de distribución, porcentaje de librerías, etc. Birkner (2013) señala este fenómeno:

Si bien esas tácticas tradicionales todavía están en juego, la era digital ha cambiado el alcance del marketing de los editores, conectando las grandes marcas de libros directamente a los usuarios finales (...) Este cambio de paradigma en las estrategias de marketing de los editores es el resultado de la creciente popularidad social y digital. Una base de consumidores orientada, así como los nuevos modelos de distribución para editores, ya que la industria del libro ahora se ha consolidado como, ante todo, un negocio orientado al comercio electrónico, y los cambios importantes resultantes en los precios y la competencia (p. 40).

Cabe aclarar, que en Argentina el precio de venta (PVP) es uniforme, pero lo que sí permite al editor es ahorrar gastos. De este modo, la relación se vuelve un poco más horizontal.

\section{Canales de comunicación offline}

De las editoriales descritas, 46 participan de la Feria Infantil y Juvenil. Este es un modo de tener visibilidad ante un público mayor, donde se permiten presentaciones o firmas de libros, dado que generalmente no se hacen presentaciones de libros infantiles como sí se hacen de adultos.

Por otro lado, el 75\% de las editoriales encuestadas hacen visitas a las escuelas o encuentros con docentes. Muchos promotores visitan a los docentes antes de comenzar el año para mostrarles no sólo manuales, sino también literatura para incluir en sus currículas. Asimismo, las editoriales y las escuelas muchas veces gestionan visitas de escritores para que los chicos puedan conversar con ellos, previo haber leído algunos de sus libros. Luego, la mayoría de ellas, tanto pequeñas como grandes, también hacen encuentros con libreros para promocionar sus novedades. 
Respecto a la prensa tradicional, el 87,5\% apuesta a este canal con envíos de cortesía a la prensa o desayunos para periodistas. Es curioso esta respuesta, dado que, como se mencionó en el capítulo anterior, la prensa no suele tener demasiado espacio para la LIJ, excepto publicaciones y programas de radio y TV especializados. Al igual que lo observado en el relevamiento del capítulo anterior, todas participan en Ferias.

Asimismo, los BBB también participan del mundo offline. Norma, SM y Penguin señalan que realizan encuentros presenciales con influencers, dentro de sus estrategias. Entre estas se encuentran invitaciones a determinados eventos como avant-premiére de películas basadas en un libro, conferencias exclusivas con determinados autores, la posibilidad de entrevistarlos, o un desayuno de influencers para anunciar el lanzamiento de un nuevo libro.

\section{Conclusiones}

La industria editorial se encuentra en plena transformación: los booktubers son legitimadores y los lectores son prosumidores. La competencia por la atención con otras industrias culturales es enorme (series, videojuegos), sumado a la sobreabundancia de libros y publicaciones. Es por ello, que un estudio sobre cómo las editoriales comunican sus libros papel y llegan a sus lectores se consideró fundamental.

De acuerdo a lo observado y documentado, y coincidiendo con los resultados de las encuestas, se pueden vislumbrar algunas tendencias.

En primer lugar, las editoriales no están donde están sus lectores. Pocas utilizan Youtube e Instagram, redes principales de los niños y jóvenes de hoy. Las editoriales pertenecientes a los grandes grupos son quienes presentan las posibilidades materiales y de recursos humanos para destinar esfuerzos a estos canales, especialmente en lo audiovisual. Algunas, hacen mejor uso de las herramientas digitales y profundizan su relación con los nuevos actores (BBBs), al realizar booktours, entrevistas a autores en redes, etc.

En segundo lugar, los influencers son hoy los agentes mediadores y prescriptores para los nativos digitales, especialmente para los adolescentes. Hay un gran potencial a desarrollar aquí para las dos editoriales analizadas y para todas las del sector. Soler (2011) dice: "Aquí ya no hay tanta diferencia entre las grandes editoriales y las pequeñas. ¿Por qué no? Porque el poder lo tiene el usuario, el lector. Él es quien va a hacer que las cosas sucedan. Hablando del libro a sus amistades, comentándolo en blogs, poniéndolo es su muro de Facebook o compartiéndolo en Twitter." Por lo tanto, acercarse a los lectores nativos digitales, con todo lo que esto implica, hablarles en su idioma, pero también escuchar sus opiniones es fundamental.

En tercer lugar, la prensa tradicional continúa siendo legitimadora en el campo literario, aún para las editoriales de LIJ que no cuentan con el espacio en los suplementos culturales que posee la literatura para adultos. Asimismo, las ferias (especialmente la FILBA) y los mediadores (bibliotecarios y docentes) también continúan como figuras de legitimación, aún ante el avance de la revolución digital. Estas formas de legitimación permite posicionar la marca editorial y son clave para el público adulto que decide la compra de los libros infantiles y juveniles. 
Por lo tanto, es fundamental incluir canales online y offline a la hora de armar una estrategia de comunicación editorial. Pero, lo más crucial para poder llegar a estar nuevas generaciones, no son tanto los canales sino crear comunidades lectoras interesadas en los libros de la editorial.

La constatación de la existencia de relaciones con influencers, al mismo tiempo de las relaciones con la prensa, a la vez que expresa la complejidad de la comunicación editorial, pone en evidencia las limitaciones de este trabajo. Abordar de manera integral la relación con estos nuevos actores requiere de la articulación de nuevos niveles de análisis y metodologías. Por lo tanto, queda abierto el interrogante para futuras investigaciones sobre las acciones que las editoriales realizan con BBBs, no sólo integrados a la comunicación como un medio más, sino como agentes activos de consumo y creación de contenidos.

\section{Notas}

1. Un ejemplo de esta columna se puede leer aquí: https://www.lanacion.com.ar/2154368que-vas-a-leer-con-tu-hijo-esta-noche-cuentos-con-monstruos-misterios-y-una-nineramagica

2. Sólo se tomaron en consideración editoriales que publican literatura infantil y juvenil, y no otro tipo de libros destinados a niños -autopublicación, religiosos, manuales, pedagógicos, comics, chistes, etc.-.

3. En este link puede verse el relevamiento: https://docs.google.com/spreadsheets/d/1w XhlAPYq3XBP-QnBURNJVP8yPSSaD0KmV0qgJ_hPL9o/edit?usp=sharing

4. En este link pueden verse las respuestas a la encuesta: https://drive.google.com/open ?id=1uInjydw0kX-L9niM58ncHbd0AZ2jzIt5qH0KG93aI4w

5. La muestra incluye respuestas de pequeñísimas editoriales como Abran Cancha, conformada por 3 personas, y de grandes editoriales como Penguin Random House con 180 empleados.

6. Recomendaciones que brinda Facebook a empresas en su sitio https://www.facebook. com/business/pages

7. En inglés en el original. Traducción propia.

8. Los libros álbum son libros en donde el texto y la imagen funcionan de manera inseparable, construyendo una historia. Es por este motivo que se considera al ilustrador como autor. Los lectores de estos libros buscan sentido a las historias teniendo en cuenta dos códigos.

9. Para un estudio más detallado sobre el tema véase Albarello, Francisco, Arri, Francisco, García, Ana Laura (2018). "Booktubers: hacia un modelo de análisis de videorreseñas bibliográficas".

10. En www.megustaleer.com hay un apartado que dice "¿Eres blogger o youtuber? Te enviamos libros para reseñar" y lleva a https://edicionanticipada.com donde se pueden inscribir y ver las reseñas de los comentadores "estrella".

11. En inglés en el original. Traducción propia. 


\section{Bibliografía}

Albarello, F.; Arri, F. y García, A. (2018). "Booktubers: nuevos jugadores en el mercado del libro". Ponencia presentada en forma parcial bajo el título "Booktubers: Entre el canon y el fandom literario", XVI Encuentro Nacional de Carreras de Comunicación (ENACOM), Universidad Nacional del Centro, Olavarría, 18 al 20 de septiembre.

Alonso-Arévalo, J. y Cordón-García, J. (2010). "El libro electrónico y los DRM”. Anuario ThinkEPI 2011.

Birkner, C. (2013). "Marketing a bestseller". Marketing News, Vol. 47, 3. Recuperado el 01/10/2018, disponible en: http://connection.ebscohost.com/c/articles/87690164/ marketing-best-seller

Conde, P. (2018). "Las estrategias del libro para dar pelea en la era de las redes". Clarín, 03 de abril. Disponible en https://www.clarin.com/cultura/estrategias-libro-dar-pelearedes_0_r11ROF-jf.html

DosDoce (2005). El papel de comunicación en la promoción cultural. Recuperado el 01/03/2018, disponible en: https://www.dosdoce.com/2006/01/31/la-comunicacionen-la-promocion-del-libro/

García Canclini, N. (2015). Hacia una antropología de los lectores. Madrid: Ariel.

Gil, M. y Rodríguez, J. (2011). El paradigma digital y sostenible del libro. Madrid: Trama Editorial.

Garcés Estrada, W.; Avitia Rodríguez, J. y Ramírez Hernández, M. (2018). "Rol que desempeña booktube en la promoción y venta de libros en México". En Ciudad, Género, Cultura y Educación en Las Regiones. México: Universidad Nacional Autónoma de México y Asociación Mexicana de Ciencias para el Desarrollo Regional A.C. Recuperado el 01/06/2018, disponible en: http://ru.iiec.unam.mx/3950/

Jobson, E. (2001). "Joining the children's cyber marketplace: A challenge for publishers". Publishing Research Quarterly. 17: 21-28. Recuperado el 01/08/2018, disponible en: https://www.researchgate.net/publication/226443323_Joining_the_children's_cyber_ marketplace_A_challenge_for_publishers

Krom, A. (2018). "Espíritu adolescente: los booktubers se vuelven aliados de los editores", La Nación, 4 de abril. Disponible en https://www.lanacion.com.ar/2122301-espirituadolescente-los-booktubers-se-vuelven-aliados-de-los-editores

Lluch, G. (2017). "Cuando los adolescentes y los jóvenes tomaron la lectura”, en Francisco Cruces Gil (ed.), ¿Cómo leemos en la sociedad digital?, Fundación Telefónica, Madrid. Recuperado el 01/06/2018, disponible en: http://www.fundaciontelefonica.com/publicaciones

Méndez, N. (2012). "Breve panorama del mercado actual de los libros infantiles y juveniles en la Argentina”. En EditadoLIJ. Recuperado el 01/01/2018, disponible en: http://editadoenlij.blogspot.com.ar/2012/07/breve-panorama-del-mercado-actual.html

Muhcina, S. y Popovici, V. (2016). "Few Aspects Regarding the Promotional Tools Used in the Marketing Activity of Publishing Houses". Ovidius University Annals, Economic Sciences Series, Ovidius University of Constantza, Faculty of Economic Sciences, vol. 0(2): 345-349. Recuperado el 29/09/2018, disponible en: https://ideas.repec.org/a/ovi/ oviste/vxviy2016i2p345-349.html 
Scherer, F. (2018). "La ola booktuber invadió la Feria del Libro", La Nación, 12 de mayo. Disponible en: https://www.lanacion.com.ar/2133853-la-ola-booktuber-invadio-laferia-del-libro

Sigal, N. (2018). “Evaluadores editoriales, ¿policías de la sensibilidad?, La Nación, 3 de noviembre. Disponible en https://www.lanacion.com.ar/2188042-evaluadores-editorialespolicias-de-la-sensibilidad

Soler, D. (2011). "Marketing editorial tradicional y online: RR.PP, promoción, web 2.0 y social media". 2ShareWorld. Recuperado el 10/01/2019, disponible en: http://www. davidsoler.es/wp-content/uploads/2011/05/Marketing\%202.0\%20editorial\%20\%20 -\%20bogota2011.pdf.

Tabernero Sala, R. (2013). "El booktrailer en la promoción de la lectura del relato". Quaderns de Filología. Estudis Literaris, vol. XVIII, pp. 211-222. Recuperado el 31/07/2018, disponible en: https://ojs.uv.es/index.php/qdfed/article/download/3302/2973

Teti, L. (2018). "Redes y prensa”. Coloquio Entre Editores. Charla inédita. Ciudad Autónoma de Buenos Aires.

Van Tassel, J. y Poe- Howfield, L. (2010). Managing Electronic Media: making, marketing, and moving digital content. EE.UU: Elsevier.

\section{Fuentes secundarias}

Fundación SM. 2017. Anuario Iberoamericano sobre el libro infantil y juvenil. Recuperado el 25/05/2018, disponible en http://www.mecd.gob.es/dms/mecd/ cultura-mecd/ areascultura/libro/mc/observatoriolect/redirige/destacados/2016/Enero/mundo-libro/ InformeSector-Enero2016/El-Sector-del-Libro-en-Espa-a---Enero-2016.pdf

Gobierno de la Ciudad de Buenos Aires. 2016. Catálogo editoriales independientes.

Recuperado el 23/06/2017, disponible en: http://www.buenosaires.gob.ar/sites/gcaba/files/ catalogo_2016.pdf

Sistema de Información Cultural de la Argentina -SINCA- (2017). Encuesta Nacional de Consumos Culturales. Recuperado el 23/03/2018, disponible en: http://camaradellibro. com.ar/informes/PDF/Encuesta-Consumos-Culturales-2017-Informe-general.pdf

\section{Anexo 1: Encuesta}

Se realizó una encuesta, enviada a todas las editoriales registradas en la CAL y en el Catálogo de Editoriales Independientes, así como a los directores de los grandes grupos, enviada por mail con un link a un Google Forms con las siguientes preguntas:

Estoy realizando mi tesis de maestría sobre "Estrategias de marketing del libro en literatura infantil y juvenil" y me sería de mucha utilidad si pudieran contestar la siguiente encuesta. Desde ya les agradezco su colaboración. 
Las siguientes preguntas se utilizarán en el marco académico de una tesis. Acepto que mencionen mi nombre y/o marca, y difusión posterior de la tesis si llegara a publicarse.

Nombre de la editorial:

Nombre de responsable (opcional)- en caso de requerir ampliar alguna respuesta-:

Mail (opcional):

1. ¿Cuántas personas conforman la editorial?

2. ¿Hay una persona resonsable de comunicación en el equipo?

Sí: ¿Externa o interna?

No

3. ¿Hay un responsable exclusivo de redes sociales (community manager)?

Sí: ¡Externa o interna?

No

4. ¿Qué canales de comunicación utilizan?

Página web

Facebook

Twitter

Instagram

Youtube

Snapchat

Whatsapp

Mailing

Otros:

5. ¿Cada cuánto actualizan el contenido de redes sociales?

Semanalmente

Diariamente

Cuando hay un nuevo lanzamiento

Nunca

6. ¿Poseen e-commerce (página web con venta directa)?

Sí

No

7. ¿Realizan pauta (anuncios) en redes sociales y web?

Facebook

Instagram

Youtube

Google Adwords

No realizamos anuncios

8. ¿Realizan acciones con influencers?

Sí

No

9. Respuesta afirmativa, ¿qué tipo de acciones?

10. ¿Con quiénes las realizan?

Niños/ jóvenes booktubers 
Mommy/ daddy bloggers

Perfiles de recomendación literaria (Por ejemplo, La gente anda leyendo)

Marcas para niños (ropa, deco u otros)

11. ¿Qué acciones offline de promoción de libros realizan?

Visitas a escuelas/ Encuentros con docentes

Visitas a librerías/ Encuentros con libreros

Desayuno de prensa

Envíos de cortesía a la prensa

Encuentro con influencers

Participación en Ferias

Otras:

12. ¿Cuál fue el libro más vendido en 2017 ?

13. ¿Qué acciones de promoción se utilizaron para dicho libro?

14. Comentarios

\section{Anexo 2}

Siguiendo un criterio organizativo, podemos dividir las editoriales en tres: pequeñas, medianas y grandes. 10 pertenecen a editoriales pequeñas, 4 a medianas y 2 a grandes. Cabe aclarar, sin embargo, que Norma (con 50 empleados) pertenece al grupo editorial transnacional Prisa, por lo que debería considerarse como un big player. Se observa que las editoriales pequeñas cuentan con 2 o 3 personas -generalmente sus propios dueños-, mientras que los grupos cuentan con más de 100 empleados.

\begin{tabular}{|l|l|l|}
\hline \multicolumn{1}{|c|}{ Chicas $<\mathbf{1 5}$} & \multicolumn{1}{|c|}{ Medianas $>\mathbf{1 5}$} & \multicolumn{1}{c|}{ Grandes $>\mathbf{1 0 0}$} \\
\hline La brujita de papel & Norma* & SM \\
\hline Abran cancha & Eduvim & Penguin Random House \\
\hline Lúdico & Hola Chicos & \\
\hline Chirimbote & El gato de hojalata (Guadal) & \\
\hline Editorial del árbol & & \\
\hline La editorial común & & \\
\hline Niño Editor & & \\
\hline Ediciones iamiqué & & \\
\hline Arte a Babor & & \\
\hline Maizal Ediciones & & \\
\hline
\end{tabular}




\begin{abstract}
In a world where the competition between cultural industries is so big (series, videogames), the communication and dissemination of books becomes central. Today bookstores are not the only sales channel, especially in the world of children's and youth books. In this work, a survey of the channels of these publishers is carried out, through the observation of their presence in social networks and their participation in book fairs. In addition, it presents the results of a survey (pilot sample, exploratory of the sector), which allows obtaining an overview of the communication strategies of children's and youth literature publishers. The work shows as a result that it is essential to include online and offline channels when putting together a communication strategy, but, the most crucial to reach new generations, is creating reading communities.
\end{abstract}

Keywords: Children's and youth literature - Editorial communication - Social networks Digital communication - Books.

Resumo: Em um mundo onde a concorrência com outras indústrias culturais é tão grande (séries, video game), a comunicação e difusão do livro tornam-se centrais. Hoje as livrarias não são o único canal de venda e, ainda menos, no mundo do livro infantil e juvenil. Neste trabalho realiza-se um relevamento dos canais de estas editoras, através da observação da sua presença em redes sociais e sua participação em feiras. Além disso, apresentam-se os resultados de uma enquete (amostra piloto, exploratória do setor), que permite obter um panorama das estratégias de comunicação das editoras de LIJ. O trabalho expõe como resultado que é fundamental incluir canais online e offline na hora de armar uma estratégia de comunicação; no entanto, o mais crucial para poder atingir a estas novas gerações é criar comunidades leitoras.

Palavras chave: Literatura infantil e juvenil - Comunicação editorial - Redes sociais - Comunicação digital - Livros.

[Las traducciones de los abstracts fueron supervisadas por el autor de cada artículo] 